\title{
Diversity of prion diseases: (no) strains attached?
}

\author{
Sebastian Brandner
}

Published online: 16 November 2010

(C) Springer-Verlag 2010

Prion diseases are neurodegenerative disorders with a remarkable variability in their clinical presentation, histopathological features and molecular pathology. Prion diseases in humans include (1) sporadic prion disease (sporadic Creutzfeldt-Jakob disease, sCJD), (2) inherited forms of prion diseases (also termed genetic or familial prion disease) such as the Gerstmann-Sträussler-Scheinker syndrome (GSS), fatal familial insomnia (FFI) or octapeptide repeat inserts (OPRI) and (3) acquired forms, such as iatrogenic CJD (iCJD), Kuru, and variant CJD (vCJD) [transmission of bovine spongiform encephalopathy (BSE) to humans]. The protein-only hypothesis postulates that the infectious agent, termed "prion" [9], is composed predominantly (if not entirely) of aggregates of misfolded, host-encoded, cellular prion protein $\left(\operatorname{PrP}^{\mathrm{C}}\right)$, commonly designated $\mathrm{PrP}^{\mathrm{Sc}}$ [15]. The partially protease resistant, misfolded ("scrapie"-) isoform $\operatorname{PrP}^{\mathrm{Sc}}$ is formed from normal ("cellular") host prion protein $\left(\mathrm{PrP}^{\mathrm{C}}\right)$ through conformational conversion. The common neuropathological features of prion diseases are a predominantly extracellular accumulation of $\operatorname{PrP}^{\mathrm{Sc}}$ in the central nervous system. Prion protein deposits can vary considerably in their pattern, distribution and intensity $[16,18]$.

More than $80 \%$ of human prion diseases manifest as sporadic CJD with an incidence of 1-2 cases per million population per year across the world, and are equally frequent in men and women. Ca. $15 \%$ of human prion diseases are associated with autosomal dominant pathogenic

\footnotetext{
S. Brandner $(\square)$

Department of Neurodegenerative Disease and Division

of Neuropathology, UCL Institute of Neurology,

London WC1N 3BG, UK

e-mail: s.brandner@ion.ucl.ac.uk;

sebastian.brandner@prion.ucl.ac.uk
}

mutations in the PRNP gene. To date, 37 pathogenic mutations and 19 non-coding polymorphisms have been described [1]. In contrast, acquired human prion diseases are rare: transmission of SCJD prions occurred through treatment with pituitary hormones pooled from human cadavers, transplantation of dura mater or cornea, the use of contaminated EEG electrodes [3], and by cannibalism among the Fore linguistic group in Papua New Guinea [2, 7]. Instead, variant CJD occurred mainly in the UK and in other countries due to human exposure to BSE prions [5], and more recently in a few cases by transmission of VCJD contaminated blood products.

A striking phenomenon of prion diseases is the existence of so-called strains. In human sporadic prion disease at least four, possibly a dozen molecular strain types (including subtypes) can be identified. The strain type is related to the clinical phenotype, the histopathological appearance, and the polymorphism on codon 129 of the human prion $(P R N P)$ gene. The molecular strain type is classified by both, fragment size and the ratio of three principal PrP bands seen after limited protease digestion. Importantly, inherited forms show a wide variability of patterns on immunoblots and often do not fit into the scheme that was originally established for SCJD, iCJD and vCJD.

The cluster "prion diseases" in this issue of Acta Neuropathologica contains both, review articles and original papers, featuring the relationship of prions with tau, inherited prion diseases, the biology of strains, processing of $\operatorname{PrP}^{\mathrm{Sc}}$ in the brain and the principle of protein misfolding cyclical amplification (PCMA).

Prion diseases share a number of features with other amyloid forming diseases of the CNS, most prominently Alzheimer's disease, but also other rare conditions such as Familial British Dementia (Worster Drought syndrome). 
The review article by Reiniger et al. [16] is a comprehensive and balanced synopsis of the amyloid cascade hypothesis and its relevance to prion disease, and it contains novel original data showing that tau hyperphosphorylation is a consistent feature of all sporadic, acquired and inherited forms of prion diseases, not only of those with formation of plaques, as was reported previously. The article also highlights the fact that PrP deposition and tau phosphorylation are strikingly congruent, and that the pattern of hyperphosphorylated tau deposits is distinct from that in Alzheimer's disease.

Two articles cover the most common forms of inherited prion diseases and one article reports a family with a novel seven octapeptide repeat insert mutation (OPRI). The review article by Capellari et al. [4] provides a summary of the most common forms of inherited prion disease. The article gives a detailed account of the glycotypes associated with E200K, D178N, V210I and 120 base pair inserts (5 OPRI). A highlight is the focus on the role of the codon 129 polymorphism on the cis (i.e. mutant) alleles in determining glycotype, histological phenotype and clinical presentation. The second contribution is specifically dedicated to the E200K mutation [13]: It complements the previous article by providing an in-depth analysis of a the phenotypic variability of the E200K mutations and sheds light on the contribution of the codon 129 genotype to the phenotype: The article also reports a co-occurrence of considerable tau (as well as $\mathrm{A} \beta$ and $\alpha$-synuclein) pathology in many E200K patients and a comparison to the article by Reiniger et al. [16] is highly recommended. A report of a family with detailed characterisation of two cases of seven OPRI (168 bp insertion) is also included in this cluster [10]. This report with excellent clinical, pathological and genetic correlation demonstrates the unique features of this disease, which is distinct from other OPRI with a striping pattern in the cerebellum $[4,16]$ and there is an obvious influence of the polymorphism on codon 129 on the clinical phenotype.

A fascinating and still largely enigmatic aspect of prion diseases are molecular strain types and their correlation to clinical and histological phenotypes. There are divergent opinions and views in the field, and we decided to invite contributions from the pioneers and leaders in the field, to provide the readership with a balanced view of the current opinions on this topic. Indeed, the three contributions highlight the difficulties to fit a highly complex biochemical phenomenon into a 15 -year old classification scheme that distinguishes four [18] or even only two $[8,14]$ types, the latter with a perplexing diversity of subtypes.
Essentially, the two different classifications describe the same migration patterns. Following limited protease digestion of CJD brain homogenates and their subsequent electrophoretic separation, typically three bands appear (Fig. 1, columns 1-4, 8, 9). The upper band consists of diglycosylated PrP and corresponds to a molecular weight of ca. $36 \mathrm{kD}$, the middle band represents monoglycosylated PrP (ca. $30 \mathrm{kD}$ ) and the lower band shows unglycosylated PrP with 19-21 kD molecular weight. One classification, originating from Collinge's group [18] describes three types of prion strains that can occur in sporadic and iatrogenic prion diseases. All three strains (Types 1-3, Fig. 1 col 1-3) show a predominant presence of the monoglycosylated band, whilst Type 4 is specific for vCJD with presence of a dominant diglycosylated band. The alternative concept originates from the laboratory of Gambetti and is described in detail in [14] and [8]. It combines two biologically distinct strains (sCJD and $\mathrm{BSE} / \mathrm{vCJD}$ ) into their type 2. To facilitate the understanding, Fig. 1 in this editorial compares the nomenclature of the London (Collinge) classification [18] with the Gambetti classification [8] and a slightly modified version provided by Kretzschmar [14].

The review article by Jeffrey et al. [11] covers scrapie in sheep and BSE in cattle, which are the most relevant sporadic prion diseases in animals. It describes in great detail the histopathological appearances and morphological variations of prion protein deposition, with an excellent diagram describing the different sub cellular localisation of PrP. An illustration highlights topology and membrane association of $\operatorname{PrP}^{\mathrm{Sc}}\left(=\mathrm{PrP}^{\mathrm{D}}\right)$. The topic "Processing $\operatorname{PrP}^{\mathrm{D}}$ in the brain" gives a comprehensive synopsis of the relevance of each cellular compartment and how they are related to PrP processing. This article is highly recommended to those interested in trafficking and processing of disease-associated PrP.

The final article by Jones et al. [12] summarises the development, applications and current limitations of a novel method to detect low levels of prion protein aggregates by PCMA, first described by the group of Soto [17] and subsequently refined or modified by others.

A number of topics had to be omitted in this cluster: we decided not to include experimental models, as there are dozens, if not hundreds of different model systems used, the phenotype of which depends on the animal species and the prion stain (e.g. RML, ME7) and numerous transgenic mice have been generated to model (often highly specifically or selectively) aspects of human prion disease. This cluster also does not contain a dedicated article on variant CJD. Instead several articles have included aspects of vCJD and compared it to sporadic and iatrogenic prion diseases. 


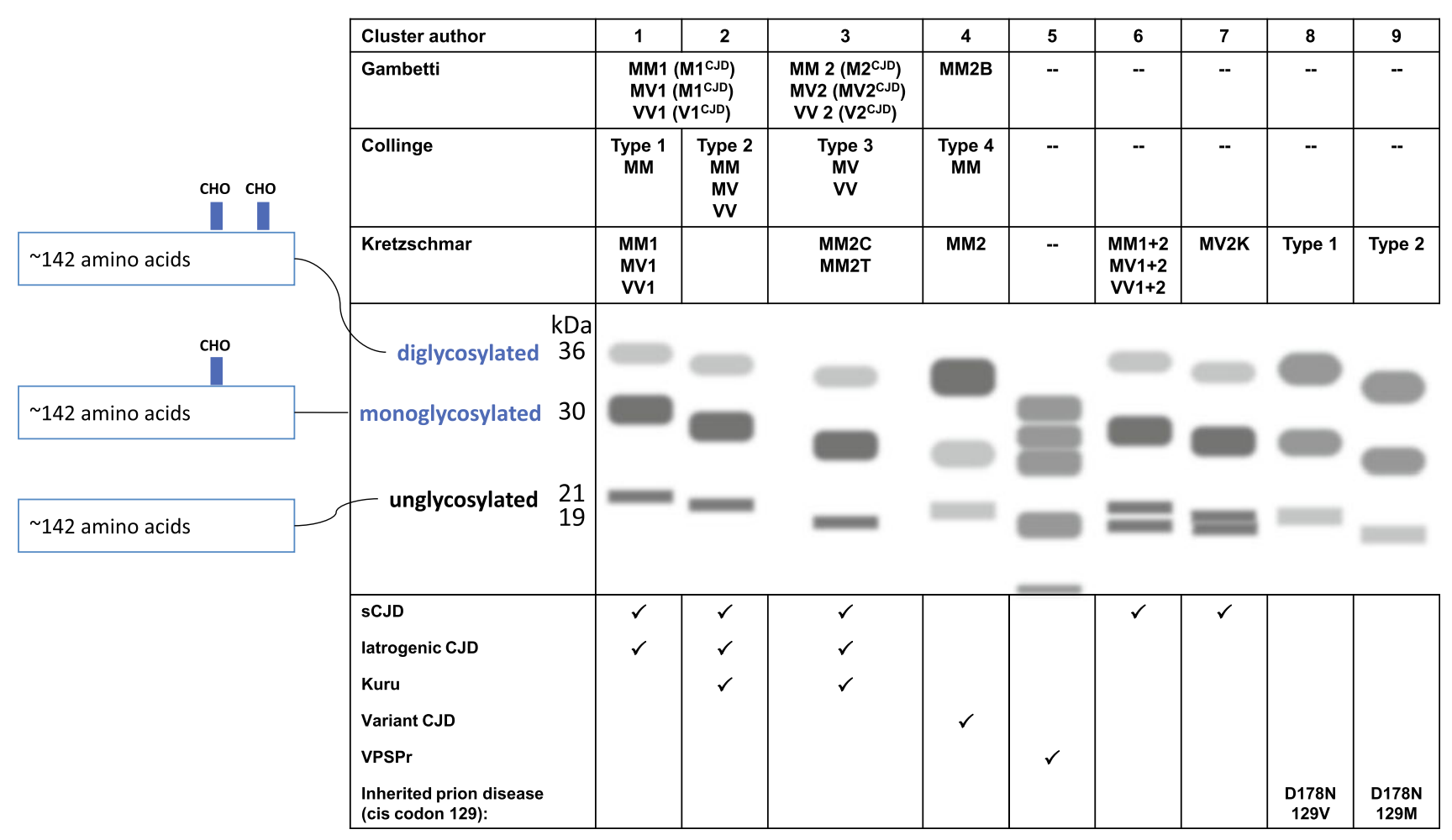

Fig. 1 Patterns of immunoblots of partially protease-digested $\mathrm{PrP}^{\mathrm{Sc}}$ in different forms of prion disease. The left part of the overview indicates how the different glycosylation forms are related to the fragment size. A precise diagram of the cleavage and the relation to the strain type is given by [8] (Figure 3). All sporadic and iatrogenic forms of prion disease are characterised by a dominance of the monoglycosylated form, designated as Types $1,2,3,[18]$ or Type 1 and 2 [14] (columns 1,2,3). The biologically distinct VCJD (and BSE) strain has a dominant diglycosylated band and hence is designated as Type $4[6,18]$ or it is designated as type $2 \mathrm{~B}$ (column 4 ).

\section{References}

1. Beck JA, Poulter M, Campbell TA, Adamson G, Uphill JB, Guerreiro R, Jackson GS, Stevens JC, Manji H, Collinge J, Mead S (2010) PRNP allelic series from 19 years of prion protein gene sequencing at the MRC Prion Unit. Hum Mutat 31:E1551-E1563

2. Brandner S, Whitfield J, Boone K, Puwa A, O'Malley C, Linehan JM, Joiner S, Scaravilli F, Calder I, Alpers MP, Wadsworth JD, Collinge J (2008) Central and peripheral pathology of kuru: pathological analysis of a recent case and comparison with other forms of human prion disease. Philos Trans R Soc Lond B Biol Sci 363:3755-3763

3. Brown P, Preece MA, Will RG (1992) "Friendly fire" in medicine: hormones, homografts, and Creutzfeldt-Jakob disease. Lancet 340:24-27

4. Capellari S, Strammiello R, Saverioni D, Kretzschmar H and Parchi P (2011) Genetic Creutzfeldt-Jakob disease and fatal familial insomnia: insights into phenotypic variability and disease pathogenesis. Acta Neuropathol. doi:10.1007/s00401-010-0760-4

5. Collinge J (1999) Variant Creutzfeldt-Jakob disease [see comments]. Lancet 354:317-323

6. Collinge J, Sidle KC, Meads J, Ironside J, Hill AF (1996) Molecular analysis of prion strain variation and the aetiology of 'new variant' CJD. Nature 383:685-690
Recently, a more refined analysis revealed that some brain regions can in fact show an overlap of two types, and accordingly are named $\mathrm{MM}(\mathrm{MV}, \mathrm{VV}) 1+2$ (columns 6, 7, compare to the single forms in columns 1 and 3 , or 2 and 3 , respectively). A new protease sensitive variant of sporadic prion disease instead shows an entirely different pattern (column 5). Inherited forms such as D178N (columns 8, 9) E200K, octarepeat inserts, etc., do not or only partially fit into this classification scheme, as they have entirely different pathobiochemical properties

7. Collinge J, Whitfield J, McKintosh E, Frosh A, Mead S, Hill AF, Brandner S, Thomas D, Alpers MP (2008) A clinical study of kuru patients with long incubation periods at the end of the epidemic in Papua New Guinea. Philos Trans R Soc Lond B Biol Sci 363:3725-3739

8. Gambetti P, Cali I, Notari S, Kong.Q, Zou W and Surewicz W (2011) Molecular biology and pathology of prion strains in human prion diseases. Acta Neuropathol. doi:10.1007/s00401-010-0761-3

9. Griffith JS (1967) Self-replication and scrapie. Nature 215: 1043-1044

10. Jansen C, Voet W, Head MW, Parchi P, Yull H, Verrips A, Wesseling P, Meulstee J, Baas F, van Gool WA, Ironside JW and Rozemuller AJ (2011) A novel seven-octapeptide repeat insertion in the prion protein gene (PRNP) in a Dutch pedigree with Gerstmann-Straussler-Scheinker disease phenotype: comparison with similar cases from the literature. Acta Neuropathol. doi: 10.1007/s00401-010-0656-3

11. Jeffrey M, McGovern G, Siso S and Gonzalez L (2011) Cellular and sub-cellular pathology of animal prion diseases: relationship between morphological changes, accumulation of abnormal prion protein and clinical disease. Acta Neuropathol. doi:10.1007/ s00401-010-0700-3

12. Jones M, Peden AH, Head MW and Ironside JW (2011) The application of in vitro cell-free conversion systems to 
human prion diseases. Acta Neuropathol. doi:10.1007/s00401010-0708-8

13. Kovacs GG, Seguin J, Quadrio I, Hoftberger R, Kapas I, Streichenberger N, Biacabe AG, Meyronet D, Sciot R, Vandenberghe R, Majtenyi K, Laszlo L, Strobel T, Budka H and Perret-Liaudet A (2011) Genetic Creutzfeldt-Jakob disease associated with the E200 K mutation: characterization of a complex proteinopathy. Acta Neuropathol. doi:10.1007/s00401-010-0713-y

14. Parchi P, Strammiello R, Giese A and Kretzschmar H (2011) Phenotypic variability of sporadic human prion disease and its molecular basis: Past, present, and future. Acta Neuropathol. doi:10.1007/s00401-010-0779-6
15. Prusiner SB (1982) Novel proteinaceous infectious particles cause scrapie. Science 216:136-144

16. Reiniger L, Lukic A, Linehan J, Rudge P, Collinge J, Mead S and Brandner S (2011) Tau, prions and Abeta: the triad of neurodegeneration. Acta Neuropathol. doi:10.1007/s00401-010-0691-0

17. Saborio GP, Permanne B, Soto C (2001) Sensitive detection of pathological prion protein by cyclic amplification of protein misfolding. Nature 411:810-813

18. Wadsworth JD and Collinge J (2011) Molecular pathology of human prion disease. Acta Neuropathol. doi:10.1007/s00401010-0735-5 